\title{
ON THE SPECTRUM OF FEIGE 7
}

\author{
D.T. Wickramasinghe \\ Royal Observatory \\ Edinburgh, U.K. \\ Brian Martin \\ Department of Applied Mathematics \\ School of General Studies \\ Australian National University \\ Canberra, Australia
}

\begin{abstract}
The Zeeman theory of hydrogen and neutral helium lines is used to analyse the spectrum of the magnetic DBA white dwarf Felge 7 . On the assumption of a centered dipole field distribution and a uniform chemical composition, the atmospheric helium to hydrogen ratio by number, $\mathrm{He}$, is found to be $\sim 10^{2}$. However, it is argued that either one, or both of these assumptions are likely to be in error.
\end{abstract}

\section{INTRODUCTION}

The majority of non-magnetic white dwarfs either have hydrogenrich atmospheres or helium-rich atmospheres. Some exceptions to this rule have been discovered, but these comprise $\leqslant 1 / 2 \%$ of all non-magnetic white dwarfs. In contrast, the proportion of stars of intermediate composition among the magnetic white dwarfs is quite high ( 20\%), and a study of these objects should provide additional information as to the physical processes which are responsible for the division of white dwarfs into the two main composition groups. Of the mixed magnetic white dwarfs, the DBA (or DAB) white dwarfs are particularly amenable to detalled study. In this paper we present an analysis of the spectrum of the DBA white dwarf Feige 7 (Liebert et al. 1977) using the Zeeman theory of hydrogen and helium (Kemic 1974).

\section{THE MODELS}

The assumptions involved and the method of computation of the present models are similar to those discussed in Martin and Wickramasinghe (1978), Wickramasinghe and Martin (1979). In addition we have now included all the neutral helium lines tabulated by Kemic (1974). These lines have been broadened assuming Voigt profiles with damping constants derfved from the tabulated results and the Stark broadening theory of neutral helium lines outlined by Griem (1974). In the absence of a 
sultable theory, we assume that the damping constants are unaltered by the presence of the magnetic field. Since at the fleld strengths of interest, magnetic broadening is dominant, this assumption does not lead to significant errors.

\section{DISCUSSION}

Llebert et al. (1977) have presented extensive observations of Feige 7. The star has a rotation period of 2.2 hrs during which the circular polarisation varies in a nearly sinusoidal manner with a mean of $\sim 0 \%$. The spectrum is also observed to vary with the same period. On the basis of the spectroscopic and polarimetric observations, these authors argue for an oblique rotator model in which the field distribution is that of a slightly off-centered dipole ( $\frac{d}{R}=0.016$ ) with the axis of rotation in the plane of the sky. From an analysis of the circular polarisation data, they also argue that multipoles higher than a simple dipole are not present with amplitudes greater than $10 \%$.

In view of the analysis by Liebert et al. (1977), we have proceeded on the assumption that the field distribution is that of a centered dipole. (Off-centering the dipole by $\frac{d}{R}=0.016$ will not change our conclusions). We assume an effective temperature $T_{e}=20,000 \mathrm{~K}$ consistent with the spectrophotometry (Greenstein 1976) and a gravity $\log (g)=8.0$. The zero fleld model atmospheres for intermediate compositions $\frac{\mathrm{He}}{\mathrm{H}}=10,10^{2}, 10^{3}, 10^{4}$ were computed as in Wickramasinghe (1979). In each case the metal abundance was taken to be $10^{-2}$ the solar value.

Theoretical spectra were computed for a range of values of the dipole field strength $B_{d}$ and the angle between the dipole axis and the IIne of sight, 1 . Since the spectrum variations are small, we compute our results with the mean spectra published by Llebert et al. (1977) and use a value of $1=90^{\circ}$. On the basis of wavelength agreement of the helium and hydrogen components with the observations, we deduce $\mathrm{B}_{\mathrm{d}}=3 \times 10^{7} \mathrm{G}$.

In figure 1 we compare the theoretical spectra for $\frac{\mathrm{He}}{\mathrm{H}}=0,10^{2}, 10^{4}$ with the observations of Liebert et al. (1977) in the spectral region $\lambda \lambda 3600-5400 \AA$. A comparison of the hydrogen-dominated (He $=0$ ) and helium dominated $\left(\frac{\mathrm{He}}{\mathrm{H}}=10^{4}\right)$ models with the observations shows the need for an atmosphere of intermediate composition with both hydrogen and helium Zeeman components in the spectrum. The model with He $=10^{2}$ appears to give the required relative strengths of the hydrogen and hellum features, and in particular of the components of $\mathrm{H}$ and HeI 4471. We emphasise that this value of the abundance is based on the assumption of a uniform composition over the stellar surface. A comparison of the theoretical spectrum for this model with the observed spectrum in the red region $(\lambda \lambda 5400-7200 A)$ is shown in figure 2 . These computations do not include HeI $\lambda 6678$ which has not been tabulated by Kemic (1974). The relative strength of the components of $\mathrm{H}_{B}$ and HeI 5876 is approximately as observed but detalled agreement is lacking. 
Figure 1: $\quad$ Observed spectra of Feige 7 (Liebert compared with these theoretical models ( $B_{d}=3 \times 10^{\prime}, 1=90^{\circ}$ ) for different atmospheric hellum contents. $0_{b}, 0_{c}, 0_{d}$ correspond to the zero intensity levels of the theoretical spectra with $\frac{\mathrm{He}}{\mathrm{H}}=100$, $\frac{\mathrm{He}}{\mathrm{H}}=10000$, He $=0$ respectively. 0 is the zero intensity level for the observed spectra. The interpolated positions of the stronger Zeeman components in a uniform fleld of $1.8 \times 10^{\prime}$ Gauss as given by Liebert et al. (1979) are also shown in the figure.

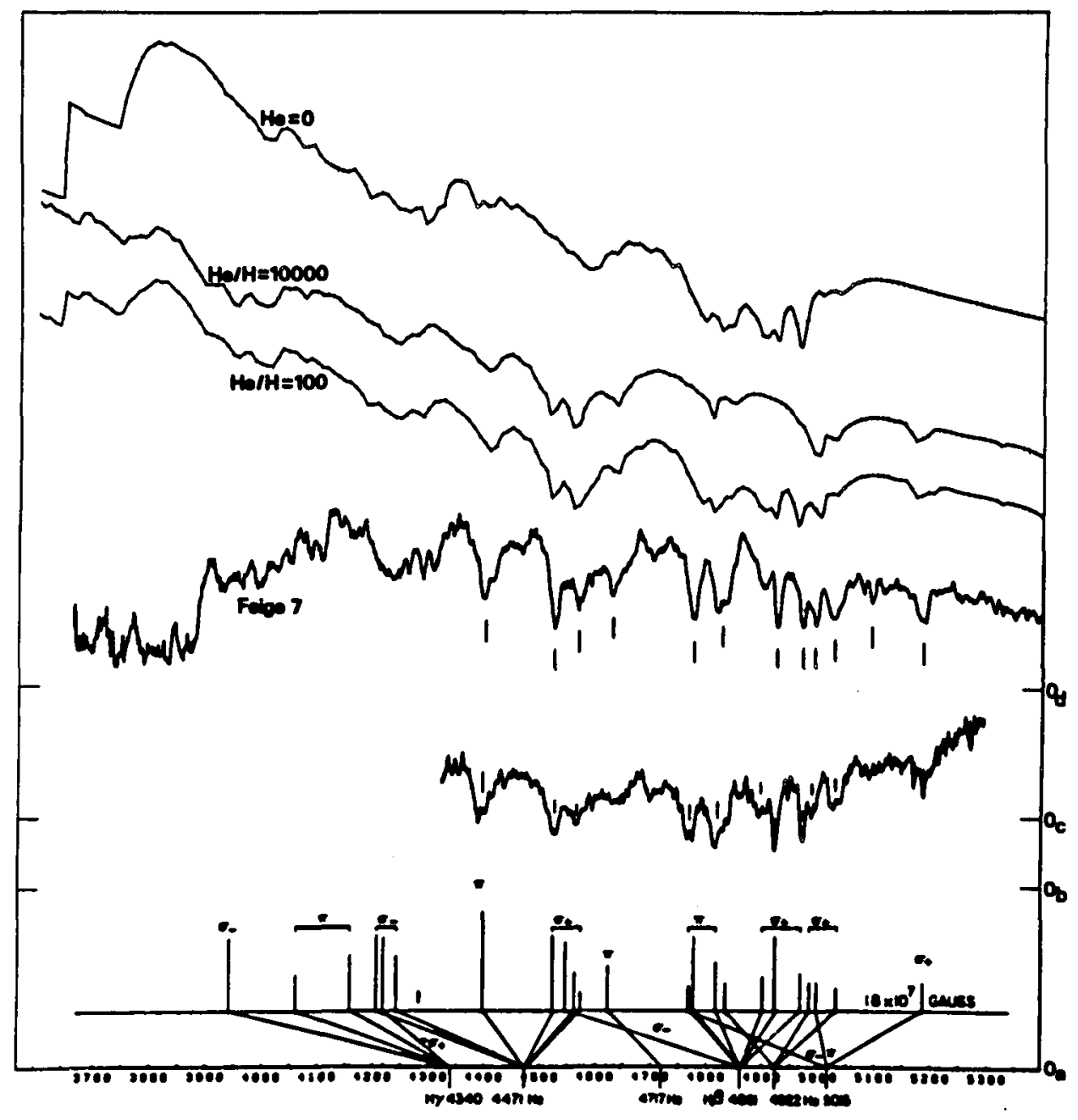


Figure 2: Comparison of the obsegved (Llebert ft al. (1977)) and the theoretical spectrum ( $B_{d}=3 \times 10^{\prime} \mathrm{G}, 1=90^{\circ}, \frac{\mathrm{He}}{\mathrm{H}}=10^{2}$ ) in the red spectral region. The zero intensity levels for the two spectra
are $0_{a}$ and $o_{b}$ respectively.

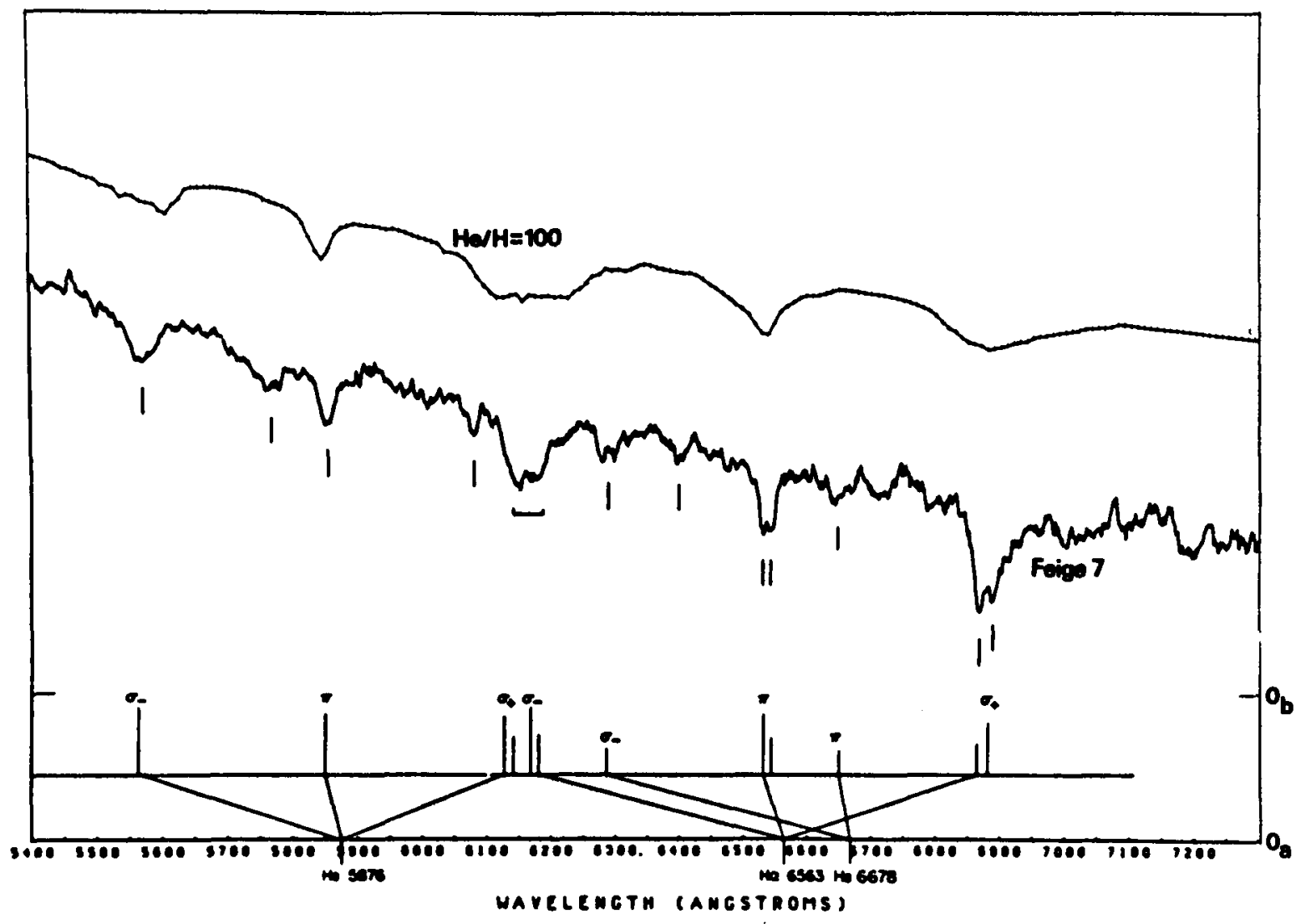


An Important result which has emerged from the present computations is the following. The field distribution of a centered dipole model does not appear to be sufficlently uniform, even when viewed equatoron $\left(1=90^{\circ}\right)$ to result in features which are as narrow as those observed in Feige 7 . This is evident from the $\sigma$ components of both the hydrogen and helium lines which are much broader than observed. Note also that the doublet structure in the region $\lambda \lambda 4750-4850$ so pronounced in the observations is not reproduced by the theory. It is important to emphasise that the lack of agreement is not due to the uncertainty in the Stark widths. Indeed a model computed with the Stark widths reduced by a factor 100 for all the hydrogen and hellum components shows no appreclable narrowing of the lines.

Our calculations appear to point to one of the following two possibilities. Either the field distribution is considerably more uniform than that of a centered dipole or our assumption that the surface composition is unfform over the stellar surface is in error. For instance the latter situation may arise if convective mixing is inhibited by the magnetic field in the equatorial region resulting in a hydrogenrich polar region and a helium-rich equatorial region. A similar situation could arise in the accretion model since accretion of hydrogenrich material would occur preferentially towards the magnetic poles. A more complete analysis of the spectrum of Feige 7 incorporating these effects is cuxrently in progress and will be discussed in detail elsewhere. For the present we merely note that the simplest models do not appear to give detalled agreement with the observations.

\section{CONCLUSIONS}

We have used the Zeeman theory of hydrogen and neutral helium lines to analyse the spectrum of Feige 7 . On the assumption of a centereddipole field distribution, and a uniform atmospheric composition we deduce $\frac{\mathrm{He}}{\mathrm{H}} \sim 10^{2}$. However there is evidence to suggest that this model is too simple. It appears either that variations in chemical composition occur across the stellar surface, or that the field distribution is considerably more uniform than that of a centered dipole.

\section{REFERENCES}

Greenstein, J. 1976, A.J., 81, 823.

Griem, H. 1974, Spectral Line Broadening by Plasmas (Academic Press). Kemic, S.B. 1974, Joint Inst. Laboratory Astrophysics Rep. 113. Liebert, J., Angel, J.R.P., Stockman, H.S., Spinrad, H. and Beaver, E.A. 1977, Ap.J., 214, 457.

Martin, B. and Wickramasinghe, D.T. 1978, M.N.R.A.S., 183, 533. Wickramasinghe, D.T. 1979 (This conference). Wickramasinghe, D.T. and Martin, B. 1979, M.N.R.A.S., (in press). 